\title{
Towards a Better Approach for Link Breaks Detection and Route Repairs Strategy in AODV Protocol
}

\author{
Saaidal Razalli Azzuhri $\mathbb{D},{ }^{1}$ Muhammad Badri Mhd Noor $\mathbb{D}{ }^{2,3}{ }^{2}$ Jafferi Jamaludin $\mathbb{D}^{\mathrm{D}}{ }^{2}$ \\ Ismail Ahmedy $\mathbb{B}^{1}{ }^{1}$ and Rafidah Md Noor ${ }^{1}$ \\ ${ }^{1}$ Department of Computer System \& Technology, Faculty of Computer Science \& Information Technology, University of Malaya (UM), \\ Kuala Lumpur, Malaysia \\ ${ }^{2}$ University of Malaya Power Energy Dedicated Advanced Centre (UMPEDAC), University of Malaya (UM), Kuala Lumpur, Malaysia \\ ${ }^{3}$ Ifcon Technology Sdn Bhd, Selangor, Malaysia \\ Correspondence should be addressed to Saaidal Razalli Azzuhri; saaidal@um.edu.my
}

Received 6 November 2017; Revised 30 January 2018; Accepted 27 February 2018; Published 10 April 2018

Academic Editor: Nathalie Mitton

\begin{abstract}
Copyright (C) 2018 Saaidal Razalli Azzuhri et al. This is an open access article distributed under the Creative Commons Attribution License, which permits unrestricted use, distribution, and reproduction in any medium, provided the original work is properly cited.
\end{abstract}

\begin{abstract}
This paper describes a parameterized approach to the Ad Hoc On-Demand Distance Vector (AODV) routing protocol using a network-simulator 2 (ns2). By utilizing two AODV's protocol functions, which are HELLO messages and local route repair, we explore the more flexible approach on these two important functions, rather than a fixed setting within the default AODV protocol. HELLO message is used to detect the broken link, while the local route repair in AODV is used to fix and discover alternative routes in the event of route failure. In this paper, two functions to optimize AODV performance have been utilized. The first is link break detection time $\left(L_{\mathrm{lb}}\right)$, using the HELLO message to detect link failure, and the second is link break position parameter $\left(L_{\mathrm{bp}}\right)$ for AODV's local route repair. The results show that the default AODV setting does not yield the best results for most defined network scenarios. In some cases, improvement compared to the default setting can be as high as $38 \%$, for local route repair strategies. This paper presents a potential flexible and parameterized approach for dealing with link breaks and route repairing strategies for AODV protocol.
\end{abstract}

\section{Introduction}

The development of routing protocols is of the important subjects within Mobile Ad Hoc Networks (MANETs), which have to deal with the typical limitations of wireless networks, including low bandwidths, high error rates, and power limitations. The main functions of MANETs protocols are to establish, maintain, and perform route repair in the event of broken links. Numerous routing protocols have been developed to cater the needs of wireless networks, or MANETs [1-3]. Such protocols can generally be categorized into two groups: reactive and proactive protocols. Reactive protocols, as the name suggests, will only find its route if it is desired by the source node. Meanwhile, proactive or tabledriven protocols will consistently maintain the routing table information at every network node [4]. In this paper, the Ad Hoc On-Demand Distance Vector (AODV) [5] protocol, one of the most widely used reactive protocols, will be studied and analysed.

In AODV, HELLO messages are the default technique used to identify broken links, while the local route repair function is used for route repair and discovery. These two functions (HELLO messages and local route repair) are very important, since AODV is a reactive type wireless protocol. They are both interconnected, since link breaks will also lead to broken routes. Therefore, the performance of AODV can be optimized through controlling these two important AODV's functions.

Prior works have been reported, dealing with link breaks detection and route repairing as means of optimizing AODV performance. Park et al. [6] reported work on HELLO inter$\mathrm{val}$, as the function of node mobility and transmission range, maximizing network throughput by using linear regression analysis. Preemptive Local Repairing Mechanism (PLRM) 
AODV, as reported in [7], takes a preventive approach to link breakage by promiscuously monitoring link quality, traffic load, and other parameters affecting network performance. The metric is used to reduce control overhead and routelength repairing processes. Another active monitoring of control message has been reported in [8], where network information is provided by the neighboring node. Scalability control in local-link repair has been demonstrated in [9], used to find alternative routes for next-to-next node, in the event of broken link. By changing the format of control packets, the network's scalability and performance have been improved, when compared to the basic AODV protocol. Another similar method has been proposed in [10], whereas the beacon-less message has been incorporated into the event of link breaks, and by only using the 802.11 b control message to reduce bandwidth utilization during route repairing. An optimized link predictability technique, based on AODV, has been demonstrated in [11]. Here the nodes estimate the link breakage time, based on the signal strength of received packets. From the received information, the affected node will decide whether to initiate local repair or new route discovery processes, much earlier than the estimated route breakage.

However, very few works have involved a comparative study of the different parameterization of HELLO messages and of local route repair functions. Presented in the following is an investigative study of the flexibility and parameterized approach of HELLO messages, and the local route repair functions in AODV. This paper's authors considered a range of different network scenarios, particularly for different levels of network load within this paper's simulation design. The simulation's first part will focus on the two important parameters, which control the periodicity of HELLO messages, specifically HELLO_INTERVAL (HI) and ALLOWED_HELLO_LOSS (AHL). The authors parameterized both $\mathrm{HI}$ and AHL with different sets of value setting and random source-destination pairs location. The second part of the simulation focuses on the local route repair functions of AODV, where a source-destination pair is fixed and is in place at both ends of the simulation area. The simulations used by this paper's authors measured the performance of the routing protocols, in terms of the Packet Delivery Ratio (PDR) metric. PDR is defined as the ratio of the total number of data packets received at the destination node, to the total number of data packets sent from the source node. The simulation results show that the PDR value can be significantly improved by setting different sets of parameters.

The rest of the paper is organized in the following way. Section 2 reviews the basic principles of the AODV protocol, and its link breaks detection mechanism and local route repair process. Section 3 discusses the implementation of the authors' parameterized concept, and the performance comparison for link breaks detection and local route repairs in AODV. This paper's conclusion and suggested future works are both presented in Section 4.

\section{Link Breaks and Route Repair Mechanism in AODV}

AODV is one of the widely used reactive type protocols in MANETs, meaning the route is initiated and maintained only when data needs to be transmitted to the destination node. The source node that serves to establish a route destination will first broadcast a special message, called the Route Request (RREQ), throughout the network. It will keep doing so, until it has been received by the node with a path to the targeted node, or if that node itself is a destination. That node provides feedback through a special message called route reply, until it reaches the source node. When the source node receives its RREP message, containing a route to the destination, it will then start to transmit the intended data to the destination node.

In AODV, a node will exchange periodic HELLO messages with its neighbor, in order to determine the link's state and to indicate that the link is still alive. During the data transmission, if any link is broken, that is it is not receiving HELLO message from the neighbor, the affected node will send a Route Error (RERR) message to all other nodes affected by the broken link, including the source node. When the source node receives the RERR message, it will begin a new route discovery process afresh. Alternatively, rather than sending a RERR message downstream, the affected node can choose a local route repair option by broadcasting a new RREQ message.

2.1. Link Breaks Detection. To determine the link health in AODV, HELLO_INTERVAL (HI) and ALLOWED_HELLO_ LOSS (AHL) are two important parameters that determine link lifetime between two neighbors. HI is defined as the time interval existing between consecutive transmissions of HELLO messages, while AHL is the parameter which determines how many HELLO message losses can be tolerated. A node that has not received a HELLO message from its neighbor after $(\mathrm{HI} \times \mathrm{AHL})$ seconds will decide that the link to its neighbor is lost, and that all other routes using that particular link will be invalidated. It is important to realize that a node should only use HELLO messages in an active route, in order to conserve bandwidth and control routing overhead. According to [5], the default setting for $\mathrm{HI}$ is 1 second, while the default setting for AHL is 2 .

The HELLO message in AODV is crucial for detecting link breaks, so that necessary actions can be taken to improve network performance. Any route dependent on that broken link will be invalidated, and the route recovery process will be activated. In MANETs, link breakage is usually caused by nodes mobility within the network and also by interference. Each node moves dynamically, in a random manner, which causes rapid change within a network. Any route that depends on the broken link will be invalidated. In particular, the $\mathrm{HI}$ value in AODV is very important for determining a network's performance. The experiment conducted in [12] has shown that a low value of $\mathrm{HI}$ increases power consumption and thereby causes the battery to be drained. This finding was based on the battery lifetime experiment. The report also shows that variations of the HI value led to significant improvements in packet delivery ratio (PDR) and end-to-end delay, while also having a minimal impact on routing overhead. In this paper, the authors investigated both AHL and HI parameters in order to determine network performance, within a different set of parameters settings. 
There will be a trade-off in setting up the AODV's AHL and HI parameter. A setting that is too low will cause a HELLO packet to suffer a collision, especially in the shared medium, and will result in the subsequent loss of HELLO packets. Adjacent nodes will "fight" to use the shared medium, and it is not always possible to transmit data packets. HELLO packets can easily suffer delays, and there is the possibility of not arriving within the stipulated time, causing a loss of connectivity. The author's motivation is based on how both parameters affect link break detection, under different network conditions.

2.2. Local Route Repairs. While detecting link breaks is crucial in AODV, the manner of dealing with link breaks is equally important in mobile networks. Basically, AODV introduces two approaches in dealing with link breakage. In the first approach, when link breakage is detected, the affected node will send a Route Error (RERR) message downwards to the source node. Upon receiving the RERR message, the source node will initiate a new route discovery process. This approach is referred to as source repair; as the name suggests, a source node will initiate a route repair and discovery process.

The second approach involves searching from the affected node itself. The affected node can set off an upstream search for the alternative route, rather than sending an RERR message back to the source. This process is called local route repairs. The affected node will initiate the discovery of a route process to the targeted node, via the RREQ message broadcast. During this process, the affected node will buffer all data projected for the destination, within the discovery period. The affected node will hold on, within the discovery period, for the route replies (RREP) message from the destination itself or any other node that has a route to the destination. Once the affected node received the RREP message, the local repair process could be considered successful, and the communication can be resumed. In AODV, the extent of the RREQ message on local repair is limited by the Time To Leave (TTL) setting. In the case of failed local repair, where no RREP message is received, the affected node will slip back to the source node for source-initiated repairs.

In AODV, the local repair scheme can reduce the time and cost of the route repairing process, which in turn improves network performance. However, in certain cases (e.g., in a failed local repair attempt), this will increase the path length and the time required for repairing the broken route. Nevertheless, an unsuccessful local repair process will result in increased network overhead, severely worsening the network's performance. The motivation of this paper's authors lies in the trade-off existing in the local repair mechanism and encouraging us to investigate the decision method regarding which scheme is best for applying various network scenarios.

As mentioned earlier, AODV supports local repair and its execution is based on certain conditions. The process depends greatly on the MAX_REPAIR_TTL parameter. This parameter indicates whether the local or the source repair process will take place, through the route repairing process. If the destination node distance is no farther than MAX_REPAIR_TTL hops away from the node where the
TABLE 1: Varying $L_{\mathrm{lb}}$ parameters.

\begin{tabular}{lcc}
\hline HELLO_INTERVAL (HI) & $\begin{array}{c}\text { ALLOWED_HELLO_LOSS } \\
\text { (AHL) }\end{array}$ & $L_{\mathrm{lb}}$ \\
\hline $0.5 \mathrm{~s}$ & 2 & $1 \mathrm{~s}$ \\
$1 \mathrm{~s}$ & 1 & $1 \mathrm{~s}$ \\
${ }^{*} 1 \mathrm{~s}$ & 2 & $2 \mathrm{~s}$ \\
$1 \mathrm{~s}$ & 3 & $3 \mathrm{~s}$ \\
$2 \mathrm{~s}$ & 2 & $4 \mathrm{~s}$ \\
\hline
\end{tabular}

${ }^{*}$ Default AODV setting.

link breaks are detected, the local repair process will be invoked. Otherwise, the source repair process will take place. In AODV, the MAX_REPAIR_TTL of AODV is set as follows:

$$
\text { MAX_REPAIR_TTL }=0.3 * \text { NET_DIAMETER. }
$$

The AODV's NET_DIAMETER value is defined as being 35 [5]. From (1), it is clear that local repair in AODV will be used if the broken link occurs in relation to the destination node, at 10 or fewer hops away. This also means that, for a small to medium-sized network, AODV will automatically decide for a local repair scheme, where the network distance is less than 11 hops. As determined from the results of this paper's simulation, this fixed local repair setting is not always the best approach. Therefore, the next section will investigate a more parameterized technique of decision-making, in choosing which approach to link breaks parameter detection, and route repair mechanism, should be invoked. The aim of this will be to improving overall network performance, by increasing PDR value.

\section{Performance Evaluation}

3.1. AODV HELLO Messages. As mentioned before, two important parameters controlling HELLO message frequency are HELLO_INTERVAL (HI) and ALLOWED_HELLO_ LOSS (AHL). HI is defined as the time interval between HELLO message transmissions. Likewise, AHL is defined as being the number of periods that the HELLO_INTERVAL can go through, without receiving a HELLO message, and a node opts that the link to its neighbor is invalid. The default value for both HI and AHL is one second and two, respectively. In this paper, the authors have defined a new parameter, called "detection of link break" time, which is as follows:

$$
\begin{aligned}
L_{\mathrm{lb}}= & \text { HELLO_INTERVAL } \\
& \times \text { ALLOWED_HELLO_LOSS. }
\end{aligned}
$$

Therefore, from $L_{\mathrm{lb}}$, link invalidation from node to its neighbor will occur if the HELLO message is not received within the duration of $L_{\mathrm{lb}}$ second. For this study's simulation, different sets of $L_{\mathrm{lb}}$ values were set when the two parameters (AHL and HI) were varied, based on Table 1.

3.1.1. Simulation Overview. This study's performance comparison was implemented using the ns2 simulator [13]. The network was designed to reflect the actual MANETs scenario. 
TABLE 2: NS-2 simulation settings.

\begin{tabular}{lc}
\hline Traffic flow & 30 flows \\
Packet size & 64 bytes \\
Maximum speed & $20 \mathrm{~m} / \mathrm{s}$ \\
802.11 MAC rate & $11 \mathrm{Mbps}$ \\
CBR source rate (packets) & 2 Kbits/s $(4$ packets $/ \mathrm{s})$ \\
Propagation model & Two-ray ground \\
Transmission range & 250 meters \\
Pause time & $0-900 \mathrm{sec}($ step of $30 \mathrm{sec})$ \\
\hline
\end{tabular}

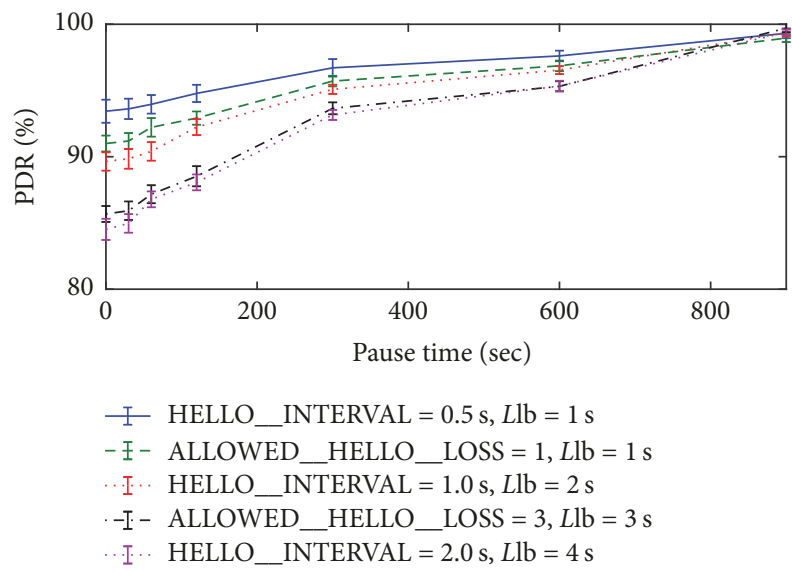

FIgURE 1: The PDR versus pause time for AODV, with different $L_{\mathrm{lb}}$.

50 mobile nodes were simulated, moving randomly in the $1500 \mathrm{~m} \times 300 \mathrm{~m}$ area dimension. A random-waypoint mobility model [14] was used, where nodes moved to a randomly selected destination, with speed homogeneously distributed from zero to MAX_SPEED. When the nodes reached the intended target, it will stop for PAUSE_TIME and after that, the same procedure will be repeated again. The generation of data traffic flows has been undertaken between pairs of nodes, in a random manner.

Packet Delivery Ratio (PDR) performance metric was computed in the simulation. PDR is the ratio of the total number of data packets received at the destination node, to the total number of data packets sent at the source node. This paper's simulation setting is presented in Table 2. 50 simulation runs were performed, each run having different mobility samples for every pause time. For more accuracy, the run results were averaged. Furthermore, a $90 \%$ confidence interval was included in the results, indicated in the graphed results. To achieve stability of the steady state conditions, traffic transmission and network trace were turned on only 300 seconds after the warming period.

3.1.2. Results and Discussions. Figure 1 shows association between the PDR performance results, and the $L_{\mathrm{lb}}$ parameter. The graph shows a decreasing PDR value for the increasing $L l b$ value. The decreasing PDR value is the result of the longer time used by the system to detect link breaks (increasing $L_{\mathrm{lb}}$ ), which was to be expected. It should be noted that, for both cases where $L_{\mathrm{lb}}$ equals $1 \mathrm{~s}$, the HI value of $0.5 \mathrm{~s}$ and the AHL

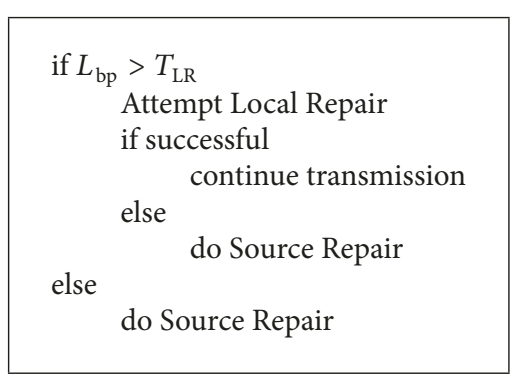

PsEudocode 1: The route repair selection scheme.

value of 2 performed better than the scenario with the HI value of $1 \mathrm{~s}$ and the AHL value of 1 . The former case has a better PDR performance, due to the AHL value of 2 requiring two consecutive HELLO message losses, before the link is marked as being broken. The letter where the AHL value is 1 will only require one HELLO message loss for the link to be invalidated.

Please note that this study's simulations have been randomly set to have a total of 30 traffic flows. There is a higher probability of the HELLO message being lost due to collisions with data packets during transmission, rather than being lost due to actual link breaks or due to mobility. In the former case the link might still be existent, here declaring the link to be broken will be unnecessary, as it will lead to poorer PDR performance.

3.2. AODV Parameterized Route Repair. As mentioned earlier in Section 3, the default AODV setting in present link breaks will only perform local repair, in the event that a broken link occurs less than 10 hops away, with the node marks being a destination. Rather than having a fixed setting or threshold used to decide which repair process is performed, this study's authors have introduced a new scheme that investigates the range of link breaks position thresholds, relative to the total network length. These ranges of thresholds will be tested through this study's designed network scenarios, in order to determine the optimal choice for the proposed work.

The proposed new parameter is called link break position parameter, $L_{\mathrm{bp}}$, presented as follows:

$$
L_{\text {bp }}=\frac{\text { link breaks hop index }}{\text { total path length }} \text {, }
$$

where $0<L_{\text {bp }} \leq 1$.

The link breaks hop index describes the position of the link breaks' location, when measured from the source node.

This study introduces another parameter, called local route repair Threshold or $T_{\mathrm{LR}}$. This parameter determines the distance position, within the end-to-end route where the link breaks happens, serving to trigger local route repair. For example, when $T_{\mathrm{LR}}$ is set as 0.5 , this simply means that if a link breakage takes place at a position which is less than halfway along total path length, the system will select a local route repair option. Otherwise, a source-initiated repair will be used. The pseudocode for the decision-making is presented in Pseudocode 1. 


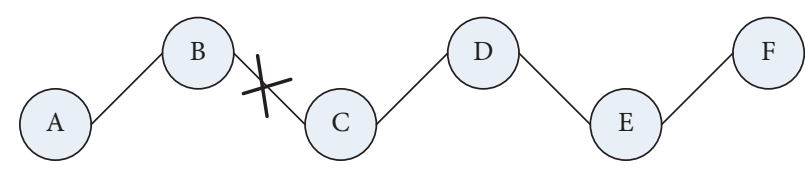

(a)

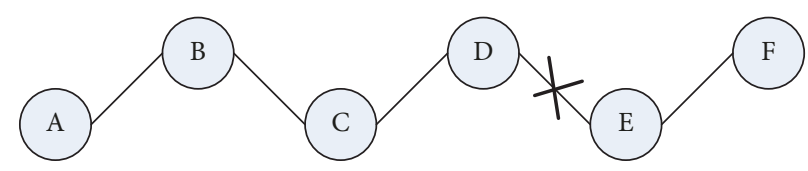

(b)

FIGURE 2: Scenarios of link breaks position, which correspond to (a) $L_{\mathrm{bp}}=0.4$ and (b) $L_{\mathrm{bp}}=0.8$.

TABLE 3: Route repair strategy $\left(T_{\mathrm{LR}}\right)$.

\begin{tabular}{lcc}
\hline$T_{\mathrm{LR}}$ & Event a & Event b \\
& $L_{\mathrm{bp}}=0.4$ & $L_{\mathrm{bp}}=0.8$ \\
\hline 0.00 & Local & Local \\
\hline 0.25 & Local & Local \\
0.50 & Source & Local \\
0.75 & Source & Local \\
1.00 & Source & Source \\
\hline
\end{tabular}

The link breaks location scenarios, for this studies' proposed scheme, are illustrated in Figures 2(a) and 2(b). The network consists of 5-hop distances between the source node (node A) and destination node (node F). In Figure 2(a), a link invalidation occurs at the hop number two (link B-C), while $L_{\mathrm{bp}}$ is $2 / 5=0.4$. In Figure 2(b)'s case, the link breaks happen at the fourth hop (between nodes D and E), and the $L_{\mathrm{bp}}$ value is $4 / 5=0.8$. For the proposed scheme, 5 different values of $T_{\mathrm{LR}}$ have been defined as $0,0.25,0.5,0.75$, and 1 .

For illustration details, Table 3 shows the decisionmaking of the proposed scheme (Pseudocode 1) for the depicted scenarios, as shown in Figure 2. This paper's authors have used all five $T_{\mathrm{LR}}$ values defined in this paper. The $T_{\mathrm{LR}}=$ 0 simply means the system will always choose a local repair, while for $T_{\mathrm{LR}}=1$, the system will always opt for source repair.

3.2.1. Simulation Overview. To test this study's proposed method, ns-2.34 was used to simulate and measure the study network's performance. The network consisted of 50 mobile nodes, randomly positioned and moving within a rectangular area measuring 1500 by $300 \mathrm{~m}^{2}$. A random-waypoint mobility model was employed [14], where mobile nodes move to the random destination at a speed homogeneously distributed from zero to MAX_SPEED. In the node speed setting, min_speed and max_speed are categorized in two sets: the first set is $[5 \mathrm{~m} / \mathrm{s}, 15 \mathrm{~m} / \mathrm{s}]$, and the second set is $[15 \mathrm{~m} / \mathrm{s}, 25 \mathrm{~m} / \mathrm{s}]$. These two sets are purposely used, so that the node average speeds are $10 \mathrm{~m} / \mathrm{s}$ for the first set and $20 \mathrm{~m} / \mathrm{s}$ for the second set.

Similarly, a constant bit rate (CBR) has been used as this simulation's traffic source. The source and destination were fixed at both ends of the simulation area, to determine the route repair method's effectiveness, as depicted in Figure 3, as the case for the one source-destination pair. Another 50 more mobile nodes are placed randomly between the source and the destination node, as mentioned earlier. The number of source-destination pairs varied from one pair to five pairs, using the CBR rates of $16 \mathrm{Kbps}$ and a packet size of 512

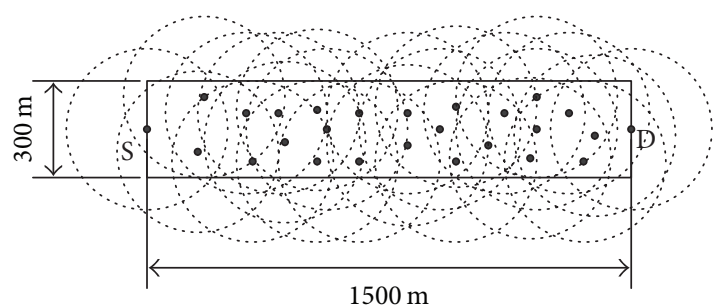

FIGURE 3: Simulation area $(\mathrm{S}=$ source, $\mathrm{D}=$ destination $)$.

TABLE 4: Simulation setting.

\begin{tabular}{lc}
\hline Name of parameters & Value(s) \\
\hline Simulation area & $1500 \mathrm{~m} \times 300 \mathrm{~m}$ \\
Nodes number & 50 \\
Local route repair threshold $T_{\mathrm{LR}}$ & $0,0.25,0.5,0.75,1.0$ \\
Average node speed & $10 \mathrm{~m} / \mathrm{s}$ and $20 \mathrm{~m} / \mathrm{s}$ \\
Mobility model & Random waypoint \\
Data traffic source rate & $16 \mathrm{Kbps}, 32 \mathrm{Kbps}$ \\
Data traffic type & Constant bit rate $(\mathrm{CBR})$ \\
Data packet size & 512 bytes \\
Source-destination pairs & $1,2,3,4,5$ \\
Range of transmission & 250 meters \\
Radio propagation model & Two-ray ground \\
802.11 MAC rate & 11 Mbps \\
Simulation runs & 30 \\
Simulation time & 900 seconds \\
\hline
\end{tabular}

bytes. With a varying number of source-destination pairs, one can also look into the method's effect when the system's traffic load was increased. The $T_{\mathrm{LR}}$ parameter was set from 0 , where local repairs are always undertaken, to 1 where source repairs are always undertaken, with intervals of 0.25 . When increasing $T_{\mathrm{LR}}$, the possibility of repairing route locally selection also decreases. For the purpose of performance evaluation, this simulation used the PDR performance metric. For accuracy and better statistical support, 30 simulations runs were performed for each defined scenario, and the results of these 30 runs were averaged. The $90 \%$ confidence intervals are also shown in the graph results. Table 4 presents the relevant parameter settings in this study's simulation.

3.2.2. Results and Discussion. Figures 4(a)-4(c) show network performance in terms of the PDR metric, using this study's proposed scheme for one-, three-, and five sourcedestination pairs, respectively, with a 16 Kbps CBR traffic flow. It has been discovered that higher node speeds have the 


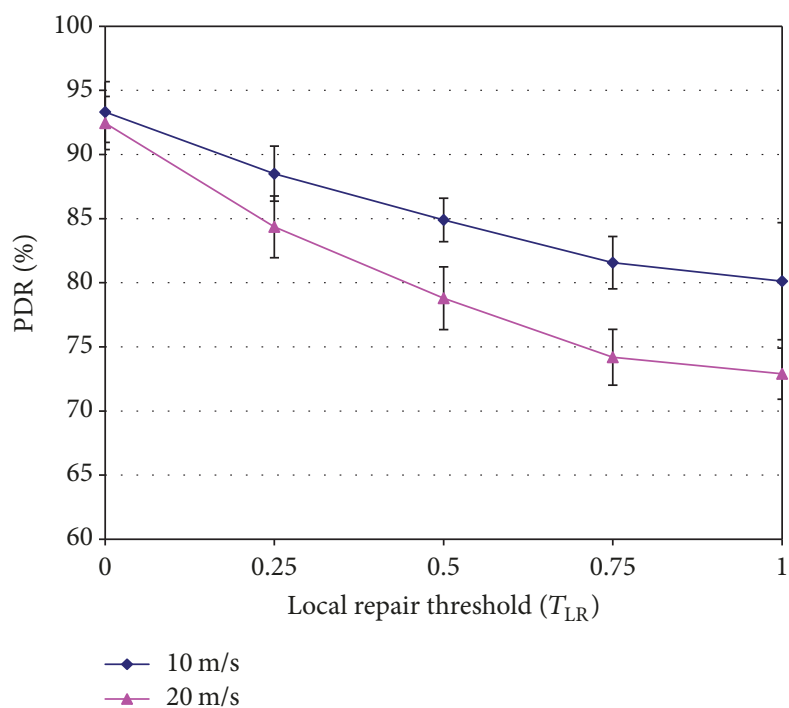

(a) One flow of $16 \mathrm{Kbps}$

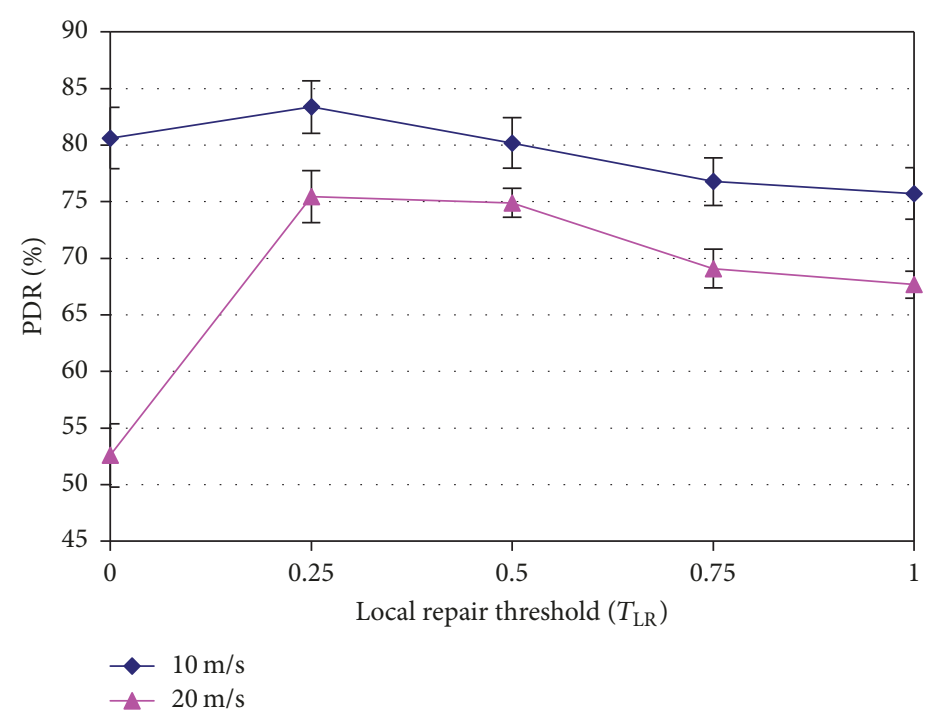

(b) Three flows of $16 \mathrm{Kbps}$

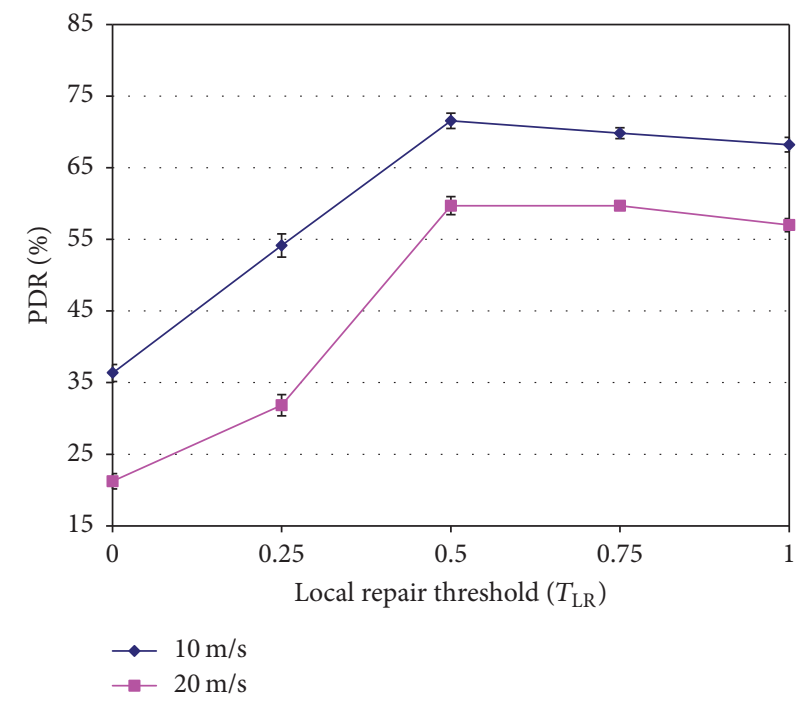

(c) Five flows of $16 \mathrm{Kbps}$ CBR

FIgURE 4: PDR versus $T_{\mathrm{LR}}$ for CBR flows of $16 \mathrm{Kbps}$.

predicted lower PDR. For varying network loads, the best performance has involved different route repair strategies, or $T_{\mathrm{LR}}$ values. For single source-destination pair results, as shown in Figure 4(a), it seems that $T_{\mathrm{LR}}=0$ (always doing local repair) achieves the highest PDR values for both the $10 \mathrm{~m} / \mathrm{s}$ and $20 \mathrm{~m} / \mathrm{s}$ node speeds. As the $T_{\mathrm{LR}}$ value increased, it was expected that there would be a decreasing trend in PDR performance. Referring to Figure 4(a), the worst PDR performance is observed when the $T_{\mathrm{LR}}=1$ (always performed source repair) and presents a significant difference when compared to $T_{\mathrm{LR}}=0$ (always do local repair). When the network flows (more load) were increased to three and five (destination pair) as depicted in Figures 4(b) and 4(c), respectively, a notable change in PDR performance was observed. Optimal $T_{\mathrm{LR}}$ values change to $T_{\mathrm{LR}}=0.25$ and $T_{\mathrm{LR}}=$ 0.5 for three and five flows, respectively.
For the results of the higher data traffic (32 Kbps traffic flow), as shown in Figures 5(a)-5(c), it was noticed that a similar results trend appeared, as presented in Figure 4. For single flow traffic shown in Figure 5(a), $T_{\mathrm{LR}}=0$ gives an optimal value for PDR performance. As the number of flows was increased to three and five, as illustrated in Figures 5(b) and 5(c), respectively, the results indicated that $T_{\mathrm{LR}}=0$ gives the worst PDR performance. For cases with three and five flows, and with both node speeds of $10 \mathrm{~m} / \mathrm{s}$ and $20 \mathrm{~m} / \mathrm{s}$, the optimum performance was observed to be $T_{\mathrm{LR}}=0.75$. Here the local route repair was invoked if it happened more than halfway along the path length, or if the link break happened near the destination node.

From these results, it seems that when there is a low network load with only one source-destination pair, as referred to in Figures 4(a) and 5(a), the packet collision is low. 


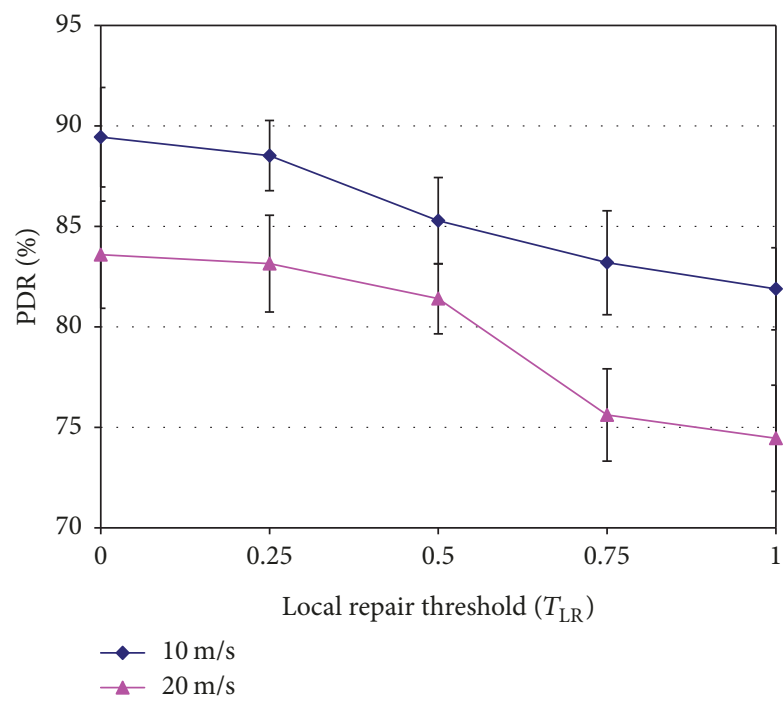

(a) One flow of $32 \mathrm{Kbps} \mathrm{CBR}$

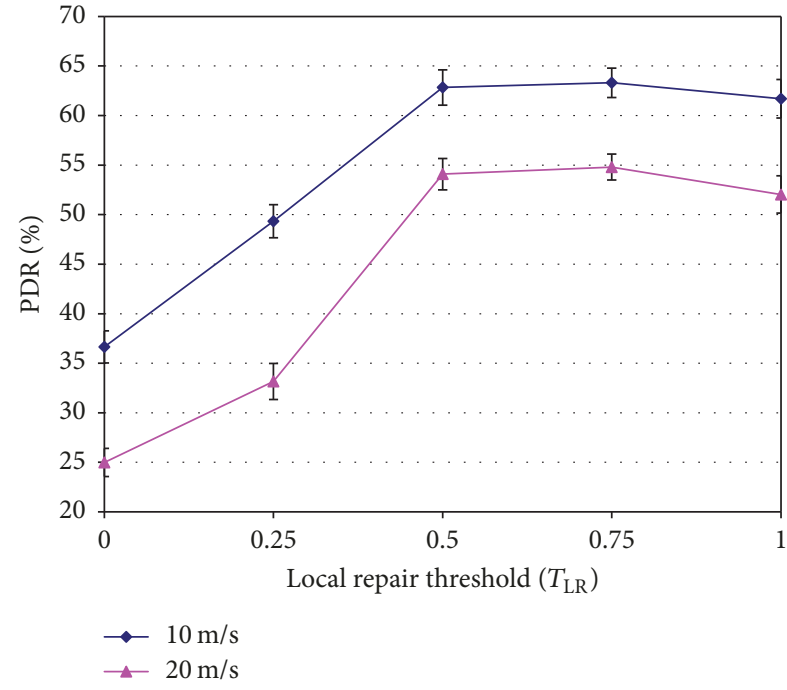

(b) Three flows of $32 \mathrm{Kbps}$ CBR

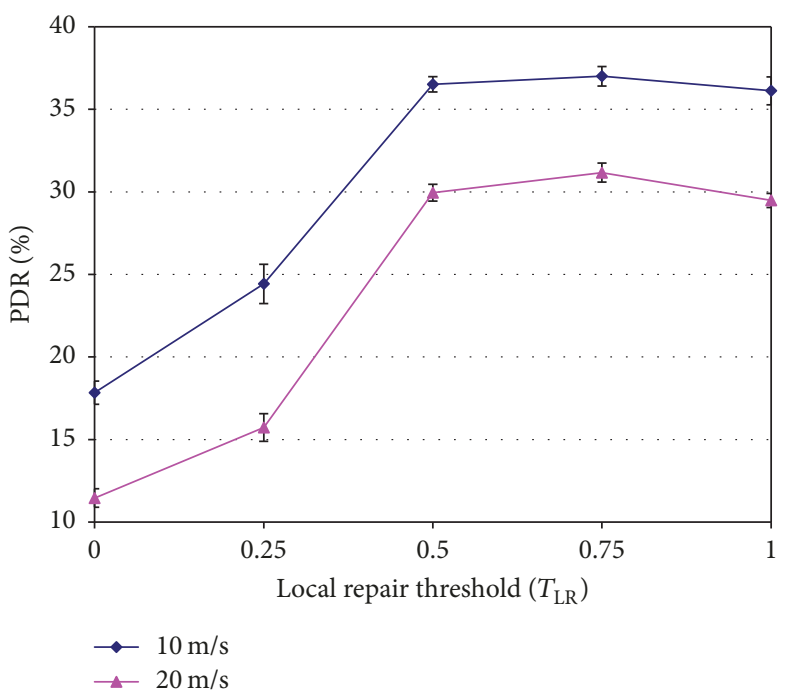

(c) Five flows of $32 \mathrm{Kbps} \mathrm{CBR}$

Figure 5: PDR versus $T_{\mathrm{LR}}$ for CBR flows of $32 \mathrm{Kbps}$.

This was indicated in the study's trace files. The route can be converged faster, if it can be repaired locally, without sending the additional control packet (RREQ and RREP) back to the source node. As the network load increases to three and five source-destination pairs, there is increased in packet loss due to collision. Additionally, the network will not scale well if a node is being forced to perform route repair locally, regardless of the 1 link breaks location. Except for Figure 4(b) (3 sourcedestination pairs with the traffic rate of $16 \mathrm{Kbps}$, where the best option is 0.25 ), optimum performance can be attained for high network loads, if the affected node is locally repaired when the link breaks occurred at halfway (0.5) from the total path length.

Note that the results shown in Figures 4 and 5 do not show any obvious connection between the node mobility speed $(10 \mathrm{~m} / \mathrm{s}$ and $20 \mathrm{~m} / \mathrm{s})$ and the $T_{\mathrm{LR}}$ optimal value. Both node speeds have very similar qualitative results. However, one can see a noticeable correlation between the network load and the $T_{\mathrm{LR}}$ optimal value, as based on the PDR results. This has been summarized in Figures 6(a) and 6(b) for the $T_{\mathrm{LR}}$ values of $0,0.25,0.5,0.75$, and 1.00 and the node speed of $10 \mathrm{~m} / \mathrm{s}$ and $20 \mathrm{~m} / \mathrm{s}$, respectively. As indicated from the results, when the network load is very low the optimum choice for $T_{\mathrm{LR}}$ is 0 , as based on the PDR value. When the network load was gradually increased, the optimal $T_{\mathrm{LR}}$ value also increased. It can be concluded that when the network load is high, to ensure efficient local route repair, the link breaks need to take place near the destination node. The simulation results also never show a $T_{\mathrm{LR}}$ of 1 , always performing source repair as the best route repair strategy.

For the purposes of further analysis, the PDR results of the optimal route repair strategy for both node speed 


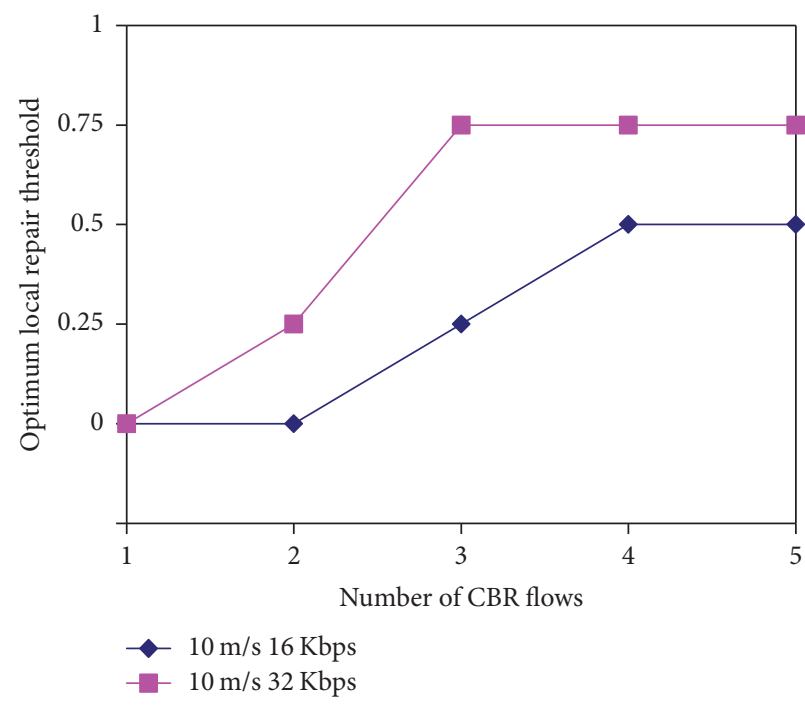

(a) $10 \mathrm{~m} / \mathrm{s}$ average node speed

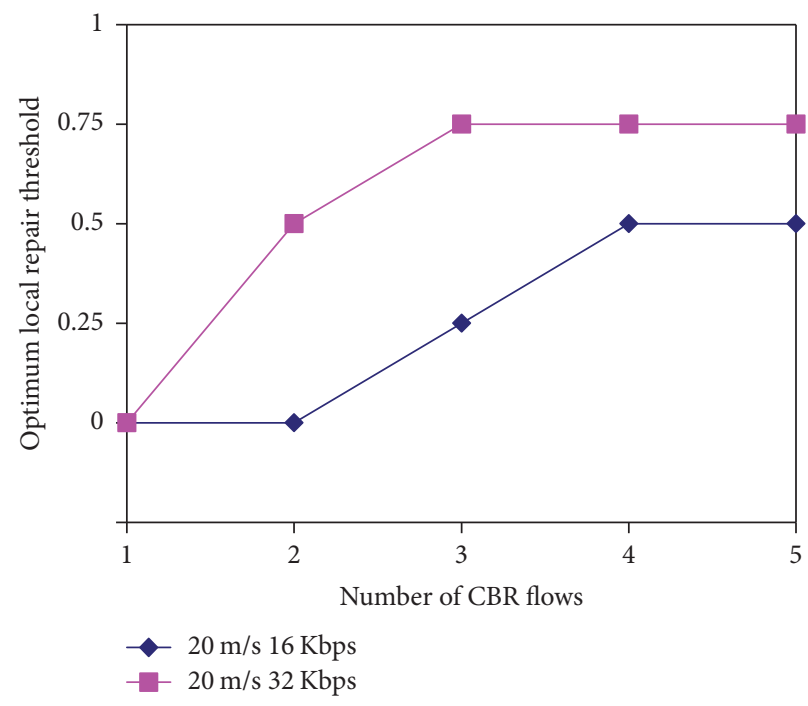

(b) $20 \mathrm{~m} / \mathrm{s}$ average node speed

FIGURE 6: $T_{\text {LR }}$ optimal for a node speed of (a) average $10 \mathrm{~m} / \mathrm{s}$ and (b) average $20 \mathrm{~m} / \mathrm{s}$.

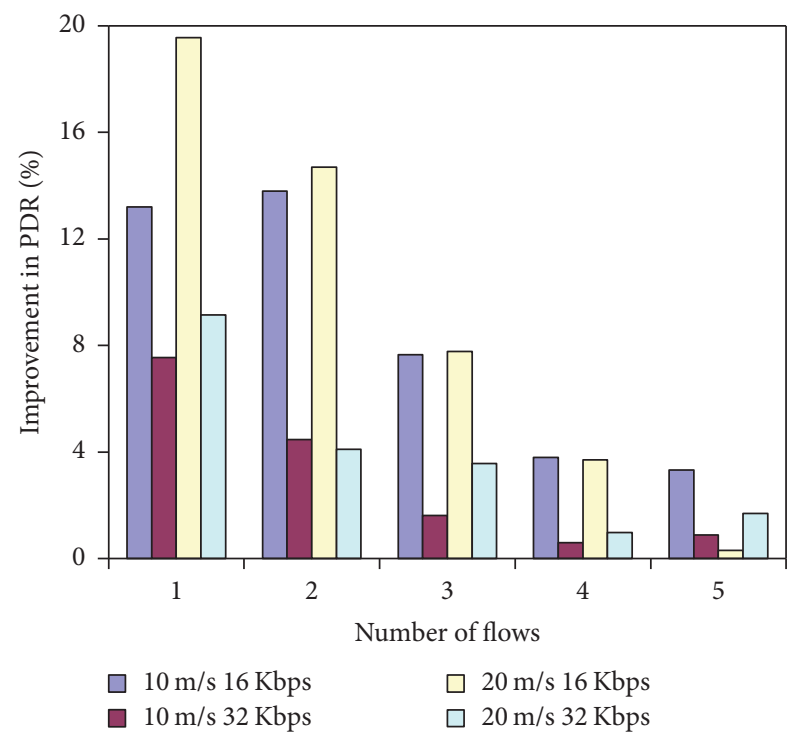

(a) $T_{\mathrm{LR}}$ optimal (PDR improvement) versus $T_{\mathrm{LR}}=1$

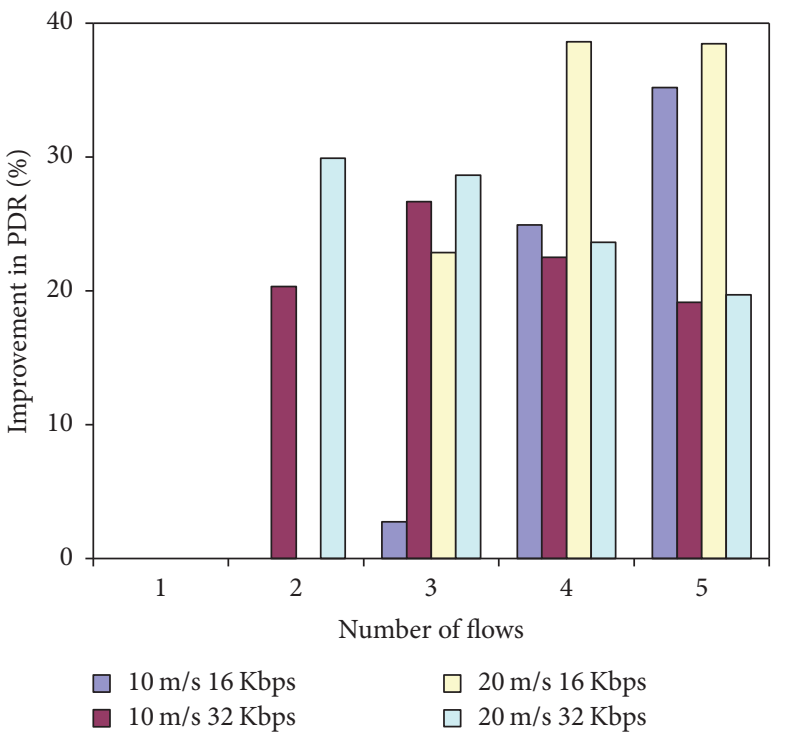

(b) $T_{\mathrm{LR}}$ optimal (PDR improvement) versus $T_{\mathrm{LR}}=0$

FIGURE 7: PDR improvements taken from the optimal choice of a route repair strategy (a) always perform source repair and (b) always perform local repair.

and network load were compared with two baseline cases, specifically the $T_{\mathrm{LR}}$ of 0 and 1 as shown in Figure 7. Figure 7 (a) shows the PDR improvements when compared to the $T_{\mathrm{LR}}$ of 1 (source-initiated repair). This always-performed source repair technique is utilized in Dynamic MANET OnDemand (DYMO) protocol, which has already changed to AODVv2 (the successor to AODV). From Figure 7(a), it was determined that the optimal repair strategy was optimized, especially when there is low network load. For instance, when there is a single flow of $16 \mathrm{Kbps}$ data traffic, with an average node speed of $20 \mathrm{~m} / \mathrm{s}$, the optimal strategy improvement over a $T_{\mathrm{LR}}$ of 1 can be reached by up to 20 percent, in terms of PDR value. As the network load or flows increased, performance improvement decreased correspondingly.

The second baseline PDR improvement was that of the $T_{\mathrm{LR}}$ of 0 (local initiated repair). The default AODV setting uses this configuration for a small- to mediumsized network [5]. Results presented in Figure 7(b) show that there is no performance gain for a low level network load, since optimal performance is achieved with a $T_{\mathrm{LR}}$ of 0 . When the network load is increased, there is significant PDR gain. The PDR improvement is more than $20 \%$ 
for most cases, with $38 \%$ being the maximum recorded gain.

From the results, it is evident that there is an inevitable need for a more flexible and adaptive route repairing method. In this paper, the conducted experiments proved that, by applying different route repairing techniques, a greater level of performance improvement can be achieved, when compared to the default setting of a wireless protocol such as AODV. Preliminary results presented here can be a base for adaptive AODV protocol. Using data such as number of nodes in network, traffic condition, traffic types, link quality, and many more network parameters, the intended protocols can utilize all this information to make a better routing decision by using regression analysis. Protocol can sense the network conditions based on the network parameters information and change the appropriate protocol parameter in order to achieve better result. This is the focus of our future works, where a lot more network parameters need to be investigated.

\section{Conclusion}

This paper presented a parameterized link breaks detection and route repairing technique for the AODV protocol. For link breaks detection, the default setting of the AODV for the HELLO message of the simulated scenario does not produce optimal results for all cases. This is especially the case in highly mobile networks. There is therefore still room for improvement. A more flexible method, concentrating on a parameterized technique, is required. Likewise, to obtain a more accurate model, more simulation is required for various network scenarios.

For the route repairing technique, this study's simulation results showed that the fixed setting of AODV, using a fixed local route repairing threshold, did not yield an optimal PDR value. It also indicated that the optimal technique depends greatly on the network load. In certain cases, there is a huge and significance improvement in using this study's defined $T_{\mathrm{LR}}$ threshold. In addition, this study compared PDR improvement with two baseline cases; the first one being the fixed threshold setting in default AODV, and the second one being the with source repair method employed by the DYMO protocol.

This paper's authors have strongly presented the cases and have argued for a more flexible and adaptive choice of both link breaks detection and route repairing technique. More simulations with various network scenarios are needed for more accurate model and to choose the best optimal results for both techniques. This study also looked at combining both detection and route repairing mechanisms, for the better improvement of AODV performance.

\section{Conflicts of Interest}

The authors declare that they have no conflicts of interest.

\section{Acknowledgments}

This work has been sponsored by the University of Malaya, under Grant no. BR002-2016.

\section{References}

[1] V. Sharma, H. Singh, M. Kaur, and V. Banga, "Performance evaluation of reactive routing protocols in MANET networks using GSM based voice traffic applications," Optik - International Journal for Light and Electron Optics, vol. 124, no. 15, pp. 2013-2016, 2013.

[2] V. K. Verma, S. Singh, and N. P. Pathak, "Analysis of scalability for AODV routing protocol in wireless sensor networks," OptikInternational Journal for Light and Electron Optics, vol. 125, no. 2, pp. 748-750, 2014.

[3] H. Simaremare, A. Abouaissa, R. F. Sari, and P. Lorenz, "Security and performance enhancement of AODV routing protocol," International Journal of Communication Systems, vol. 28, no. 14, pp. 2003-2019, 2015.

[4] E. M. Royer and C.-K. Toh, "A review of current routing protocols for ad hoc mobile wireless networks," IEEE Personal Communications, vol. 6, no. 2, pp. 46-55, 1999.

[5] C. Perkins, E. Belding-Royer, and S. Das, "Ad hoc on-demand distance vector (AODV) routing," 2070-1721, 2003.

[6] N.-U. Park, J.-C. Nam, and Y.-Z. Cho, "Impact of node speed and transmission range on the hello interval of MANET routing protocols," in Proceedings of the 2016 International Conference on Information and Communication Technology Convergence, ICTC 2016, pp. 634-636, October 2016.

[7] Z. Zhang, Z. Li, and J. Chen, "An enhanced AODV route repairing mechanism in wireless ad-hoc sensor network," Journal of Communications, vol. 9, no. 8, pp. 651-657, 2014.

[8] J.-S. Liu and C.-H. R. Lin, "RBR: Refinement-based route maintenance protocol in wireless ad hoc networks," Computer Communications, vol. 28, no. 8, pp. 908-920, 2005.

[9] J. Jain, R. Gupta, and T. K. Bandhopadhyay, "Scalability enhancement of AODV using local link repairing," International Journal of Electronics, vol. 101, no. 9, pp. 1230-1243, 2014.

[10] J. Jain, R. Gupta, and T. K. Bandhopadhyaya, "Performance analysis of proposed local link repair schemes for ad hoc on demand distance vector," IET Networks, vol. 3, no. 2, pp. 129136, 2014.

[11] A. Yadav, Y. N. Singh, and R. R. Singh, "Improving Routing Performance in AODV with Link Prediction in Mobile Adhoc Networks," Wireless Personal Communications, vol. 83, no. 1, pp. 603-618, 2015.

[12] C. Gomez, M. Catalan, X. Mantecon, J. Paradells, and A. Calveras, "Evaluating performance of real ad-hoc networks using AODV with hello message mechanism for maintaining local connectivity," in Proceedings of the 2005 IEEE 16th International Symposium on Personal, Indoor and Mobile Radio Communications, PIMRC 2005, pp. 1327-1331, September 2005.

[13] T. Issariyakul and E. Hossain, Introduction to Network Simulator NS2, Springer Science \& Business Media, 2011.

[14] C. Bettstetter, G. Resta, and P. Santi, "The node distribution of the random waypoint mobility model for wireless ad hoc networks," IEEE Transactions on Mobile Computing, vol. 2, no. 3, pp. 257-269, 2003. 


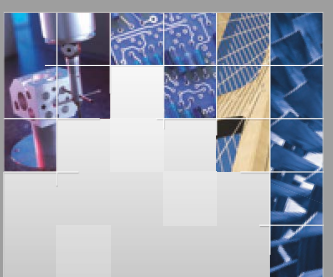

\section{Enfincering}
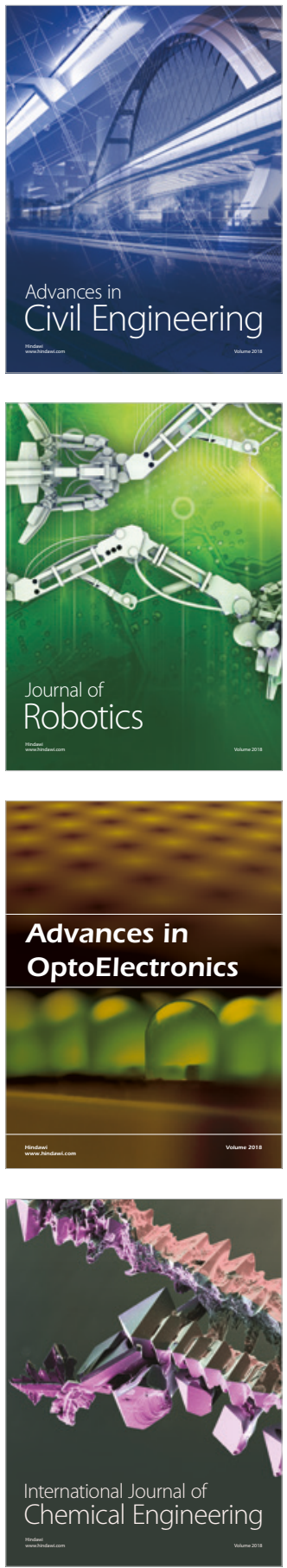

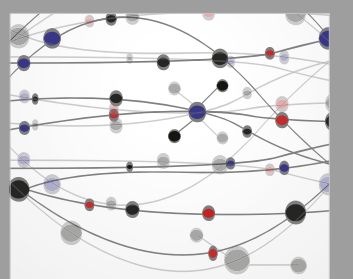

\section{Rotating \\ Machinery}

The Scientific World Journal

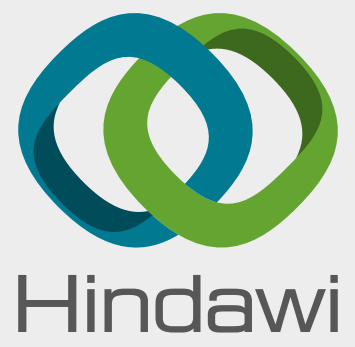

Submit your manuscripts at

www.hindawi.com
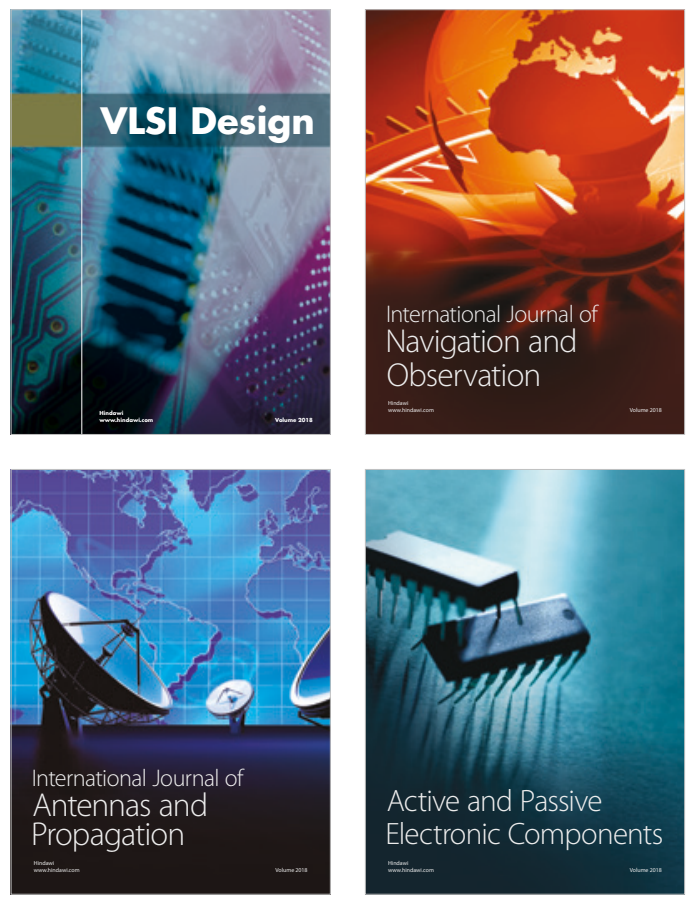
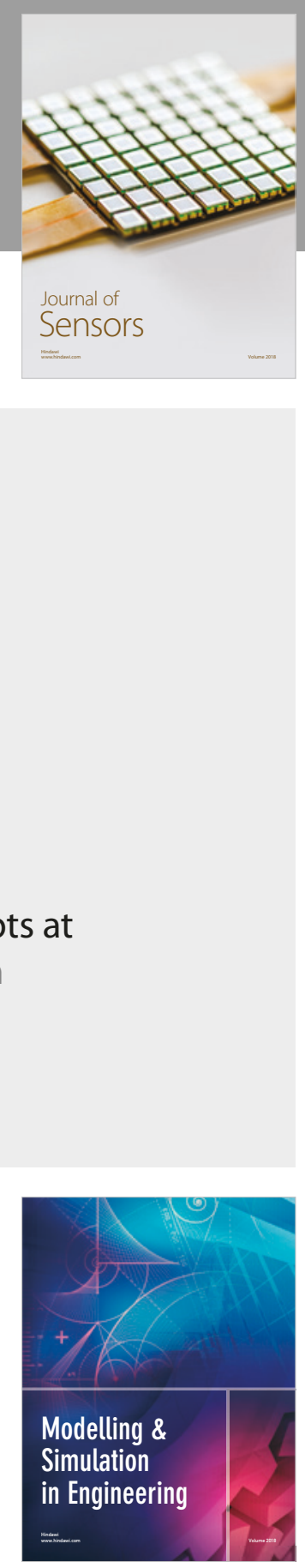

\section{Advances \\ Multimedia}
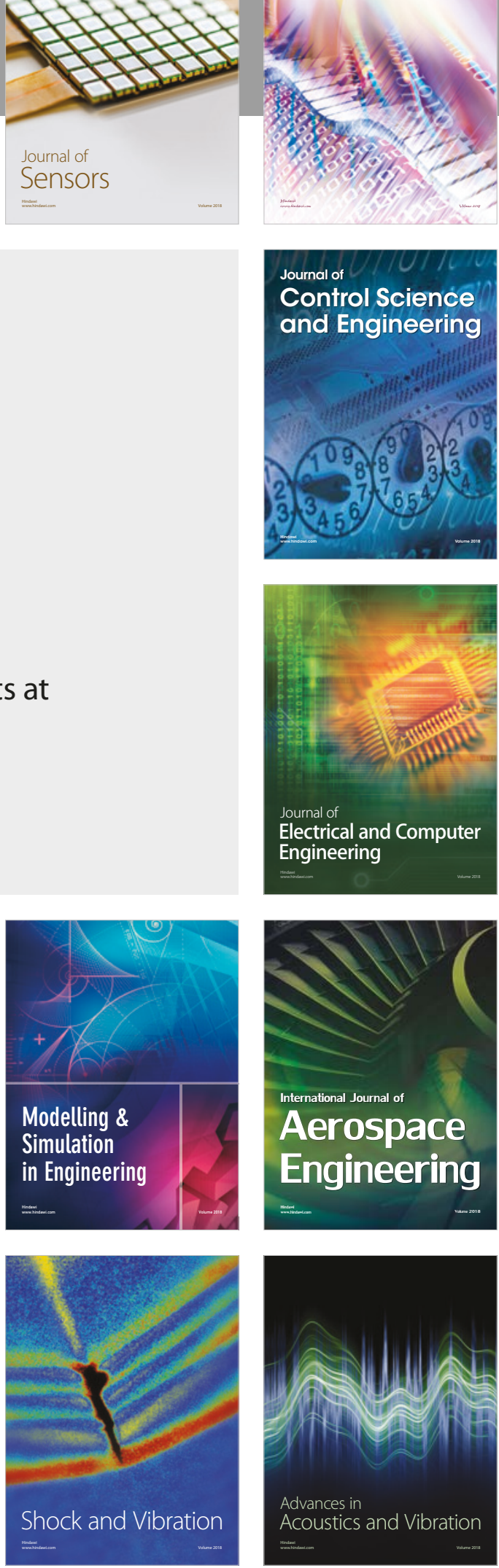